Eitan Tadmor · Dongming Wei

\title{
On the global regularity of subcritical Euler-Poisson equations with pressure
}

Received September 8, 2006 and in revised form May 8, 2007

\begin{abstract}
We prove that the one-dimensional Euler-Poisson system driven by the Poisson forcing together with the usual $\gamma$-law pressure, $\gamma \geq 1$, admits global solutions for a large class of initial data. Thus, the Poisson forcing regularizes the generic finite-time breakdown in the $2 \times 2 p$-system. Global regularity is shown to depend on whether or not the initial configuration of the Riemann invariants and density crosses an intrinsic critical threshold.
\end{abstract}

Keywords. Euler-Poisson equations, Riemann invariants, critical thresholds, global regularity

\section{Introduction}

It is well known that the systems of Euler equations for compressible flows can and will break down at a finite time even if the initial data are smooth. A prototype example for such systems is provided by the $2 \times 2$ system of isentropic gas dynamics

$$
\left\{\begin{array}{l}
\rho_{t}+(\rho u)_{x}=0, \\
(\rho u)_{t}+\left(\rho u^{2}\right)_{x}=-p_{x},
\end{array}\right.
$$

where the pressure $p=p(\rho)$ is given by the usual $\gamma$-law, $p(\rho)=A \rho^{\gamma}$. By using the method introduced in [La64] to deal with pairs of conservation laws, it can be shown that 1.1 will lose the $C^{1}$-smoothness due to the appearance of shock discontinuities unless its two Riemann invariants are nondecreasing. Thus, the finite-time breakdown of (1.1) is generic in the sense that it holds for all but a "small" set of initial data.

On the other hand, if we replace the pressure by Poisson forcing, then we arrive at the system of Euler-Poisson equations

$$
\left\{\begin{array}{l}
\rho_{t}+(\rho u)_{x}=0, \\
(\rho u)_{t}+\left(\rho u^{2}\right)_{x}=-k \rho \varphi_{x}, \quad k>0
\end{array}\right.
$$

subject to initial data $\left(u_{0}, \rho_{0}>0\right)$. Here $\varphi=\varphi(\rho)$ is the potential, which is dictated by the (one-dimensional) Poisson equation, $\varphi_{x x}=-\rho$. In this case, there is a "large" set of

E. Tadmor, D. Wei: Department of Mathematics, Institute for Physical Science and Technology and Center of Scientific Computation and Mathematical Modeling (CSCAMM),

University of Maryland, College Park, MD 20742, USA;

e-mail: tadmor@cscamm.umd.edu,dwei@cscamm.umd.edu

Mathematics Subject Classification (2000): 35Q35, 35B30 
initial configurations which yield global smooth solutions. More precisely, [ELT01] have shown that $(1.2)$ admits a global smooth solution if and only if

$$
u_{0 x}(x)>-\sqrt{2 k \rho_{0}(x)} .
$$

Thus, following the terminology of [LT02], the curve $u_{0 x}+\sqrt{2 k \rho_{0}}=0$ is a "critical threshold" in configuration space which separates between initial configurations leading to finite-time breakdown and a "large" set of subcritical initial configurations which yield global smooth solutions. In particular, (1.3) allows negative velocity gradients (depending on the local amplitude of the density), which otherwise are excluded in the case of inviscid Burgers equations, corresponding to $k=0$.

In this paper we turn our attention to the full Euler-Poisson equations driven by both pressure and Poisson forcing,

$$
\left\{\begin{array}{l}
\rho_{t}+(\rho u)_{x}=0 \\
(\rho u)_{t}+\left(\rho u^{2}\right)_{x}=-p(\rho)_{x}-k \rho \varphi_{x}, \quad k>0 \\
-\varphi_{x x}=\rho
\end{array}\right.
$$

These equations govern different phenomena, ranging from the largest scale of, e.g., the evolution gravitational collapse in stars, to applications in the smallest scale of, e.g., semiconductors. There is a considerable amount of literature available on the local and global behavior of Euler-Poisson and related problems. Consult [Ma86] for local existence in the small $H^{s}$-neighborhood of a steady state of self-gravitating stars, [CW96] for global existence of weak solutions with geometrical symmetry, [Gu98] for global existence for 3-D irrotational flow, [MN95] for isentropic case, and [JR00], [PRV95] for isothermal case. Consult [Pe90], [MP90], [Si85], [En96], [WC98], [BW98], and in particular [En96] (more about that below), for nonexistence results and singularity formation. The question of global smoothness vs. finite breakdown was studied in a recent series of works of Engelberg, Liu and Tadmor, in terms of a critical threshold phenomena for 1-D "pressure-less" Euler-Poisson equations [ELT01] and 2-D restricted Euler-Poisson equations [LT02, LT03].

The natural question that arises in the present context of full Euler-Poisson equations 1.4 is whether the pressure enforces a generic finite-time breakdown, or whether the presence of Poisson forcing preserves global regularity for a "large" set of initial configurations. We answer this question of "competition" between pressure and Poisson forcing, proving that the Euler-Poisson equations (1.4) with $\gamma \geq 1$ admit global smooth solutions for a "large" set of subcritical initial data such that

$$
u_{0 x}(x)>-K_{0} \sqrt{\rho_{0}(x)}+\sqrt{A \gamma} \frac{\left|\rho_{0 x}(x)\right|}{\rho_{0}(x)^{(3-\gamma) / 2}}, \quad \gamma \geq 1 .
$$

Here, $K_{0}$ is a constant depending on $k, \gamma$ and the initial data. In the particular (and important) case of isothermal equations, $\gamma=1$, we have $K_{0}=\sqrt{2 k}$ and $(1.5)$ amounts to a sharp critical threshold,

$$
u_{0 x}(x) \geq-\sqrt{2 k \rho_{0}(x)}+\sqrt{A} \frac{\left|\rho_{0 x}(x)\right|}{\rho_{0}(x)}, \quad \gamma=1 .
$$


The inequalities (1.5), 1.6 quantify the competition between the destabilizing pressure effects, as the range of subcritical initial configurations shrinks with the growth of the amplitude of the pressure, $A$, while the stabilizing effect of the Poisson forcing increases the subcritical range with a growing $k$. In particular, (1.6) with $A=0$ recovers the pressurefree critical threshold (1.3).

Formation of singularities and global regularity of (1.4) were addressed earlier by Engelberg in [En96]. His results show finite-time breakdown if the quantity $u_{0 x}(x)-$ $\sqrt{A \gamma}\left|\rho_{0 x}(x)\right| \rho_{0}(x)^{(\gamma-3) / 2}$ is "sufficiently negative" at some point. Our contribution here is to quantify the critical threshold behind this asymptotic statement. To fully appreciate this quantified threshold, we turn to the converse statement in [En96, Theorem 2]: it asserts the global regularity of (1.4) for a class of initial data such that $u_{0 x}(x)-$ $\sqrt{A \gamma}\left|\rho_{0 x}(x)\right| \rho_{0}(x)^{(\gamma-3) / 2}>0$. It is a "nongeneric" class (in the sense of requiring both Riemann invariants at $t=0$ to be globally increasing). In fact, by (1.5) one has a negative threshold, $-K_{0} \sqrt{\rho_{0}}$, implying the existence of a "large" class of subcritical initial data with global regularity.

The paper is organized as follows. In Section 2, we reformulate the system (1.4) with its Riemann invariants as a preparation for the analysis carried out in Sections 3 and 4 . In Section 3, we prove our main results, providing sufficient conditions for "large" sets of subcritical initial configurations which yield global smooth solution. In Section 4 , we give examples of finite-time breakdown for supercritical initial data. Combining our results in Sections 3 and 4, they confirm the existence of a critical threshold phenomena for the full Euler-Poisson equations (1.4).

\section{Riemann invariants}

\subsection{The Euler-Poisson equations with $\gamma$-law pressure: $\gamma>1$}

We begin by rewriting the Euler-Poisson equations (1.4) as a first order quasilinear system

$$
\left(\begin{array}{l}
\rho \\
u
\end{array}\right)_{t}+J\left(\begin{array}{l}
\rho \\
u
\end{array}\right)_{x}=\left(\begin{array}{l}
0 \\
-k \varphi_{x}
\end{array}\right),
$$

where the Jacobian $J:=\left(\begin{array}{cc}u & \rho \\ A \gamma \rho^{\gamma-2} & u\end{array}\right)$ has two different eigenvalues

$$
\lambda:=u-\sqrt{A \gamma} \rho^{(\gamma-1) / 2}<\mu:=u+\sqrt{A \gamma} \rho^{(\gamma-1) / 2},
$$

and let $R$ and $S$ denote the Riemann invariants of the corresponding Euler system (1.1),

$$
R:=u-\frac{2 \sqrt{A \gamma}}{\gamma-1} \rho^{(\gamma-1) / 2} \quad \text { and } \quad S:=u+\frac{2 \sqrt{A \gamma}}{\gamma-1} \rho^{(\gamma-1) / 2} .
$$

They satisfy the system of equations

$$
\begin{aligned}
R_{t}+\lambda R_{x} & =-k \varphi_{x}, \\
S_{t}+\mu S_{x} & =-k \varphi_{x},
\end{aligned}
$$


coupled through the Poisson equation $-\phi_{x x}=\rho$. If we set $r:=R_{x}, s:=S_{x}$ then upon differentiation of 2.3 we get

$$
\begin{gathered}
r_{t}+\lambda r_{x}+\lambda_{S} r s+\lambda_{R} r^{2}=k \rho, \\
s_{t}+\mu s_{x}+\mu_{S} s^{2}+\mu_{R} r s=k \rho .
\end{gathered}
$$

Next, we observe that $\lambda=\frac{R+S}{2}-\frac{\gamma-1}{4}(S-R)$ and $\mu=\frac{R+S}{2}+\frac{\gamma-1}{4}(S-R)$. Hence, expressed in terms of $\theta:=(\gamma-1) / 2$, we have, for $\gamma \geq 1$,

$$
\lambda_{R}=\mu_{S}=\frac{1+\theta}{2}, \quad \lambda_{S}=\mu_{R}=\frac{1-\theta}{2}, \quad \theta:=\frac{\gamma-1}{2} \geq 0,
$$

and the pair of equations 2.4 is recast into the form

$$
\begin{aligned}
& r^{\prime}+\frac{1+\theta}{2} r^{2}+\frac{1-\theta}{2} r s=k \rho, \\
& s^{\prime}+\frac{1+\theta}{2} s^{2}+\frac{1-\theta}{2} r s=k \rho .
\end{aligned}
$$

Here and below, \{\}$^{\prime}:=\partial_{t}+\lambda \partial_{x}$ and \{\}$^{\prime}:=\partial_{t}+\mu \partial_{x}$ denote differentiation along the $\lambda$ and $\mu$ particle paths,

$\Gamma_{\lambda}:=\{(x, t) \mid \dot{x}(t)=\lambda(\rho(x, t), u(x, t))\}, \quad \Gamma_{\mu}:=\{(x, t) \mid \dot{x}(t)=\mu(\rho(x, t), u(x, t))\}$.

To continue, we rewrite the equation for $\rho$ as

$$
\left(\rho_{t}+\lambda \rho_{x}\right)+\frac{\mu-\lambda}{2} \rho_{x}+\rho \frac{s+r}{2}=0 .
$$

Since $s-r=S_{x}-R_{x}=2 \sqrt{A \gamma} \rho^{(\gamma-3) / 2} \rho_{x}$, it enables us to express

$$
\frac{\mu-\lambda}{2} \rho_{x}=\sqrt{A \gamma} \rho^{(\gamma-1) / 2} \rho_{x}=\rho \frac{s-r}{2},
$$

so that the $\rho$ equation 2.6) can be written along the $\lambda$ particle path as $\rho^{\prime}+\rho s=0$. Similarly, it can be written along the $\mu$ particle path as $\rho^{\prime}+\rho r=0$. Assembling the above equations together, we arrive at the following system governing $r, s$ and $\rho$ :

$$
\left\{\begin{array}{l}
r^{\prime}+\frac{1+\theta}{2} r^{2}+\frac{1-\theta}{2} r s=k \rho, \\
\rho^{\prime}+\rho s=0,
\end{array}\right.
$$

and

$$
\left\{\begin{array}{l}
s^{\prime}+\frac{1+\theta}{2} s^{2}+\frac{1-\theta}{2} r s=k \rho, \\
\rho^{\prime}+\rho r=0 .
\end{array}\right.
$$

Finally, we use the integration factors $1 / \sqrt{\rho}$ and $r / 2 \rho \sqrt{\rho}$ in the first and second equations of each pair in 2.7), to conclude

$$
\begin{aligned}
& \left(\frac{r}{\sqrt{\rho}}\right)^{\prime}+\frac{1+\theta}{2} \frac{r^{2}}{\sqrt{\rho}}-\frac{\theta}{2} \frac{r s}{\sqrt{\rho}}=k \sqrt{\rho}, \\
& \left(\frac{s}{\sqrt{\rho}}\right)^{\prime}+\frac{1+\theta}{2} \frac{s^{2}}{\sqrt{\rho}}-\frac{\theta}{2} \frac{r s}{\sqrt{\rho}}=k \sqrt{\rho} .
\end{aligned}
$$




\subsection{The isothermal case $\gamma=1$}

In this case, the two eigenvalues are $\lambda=u-\sqrt{A}<\mu=u+\sqrt{A}$ with the corresponding Riemann invariants $R=u-\sqrt{A} \ln \rho$ and $S=u+\sqrt{A} \ln \rho$. Their derivatives, $r$ and $s$, satisfy the pair of equations, corresponding to $2.8 \mathrm{a}$, , 2.8b with $\theta=(\gamma-1) / 2=0$,

$$
\begin{aligned}
& \left(\frac{r}{\sqrt{\rho}}\right)^{\prime}+\frac{1}{2} \frac{r^{2}}{\sqrt{\rho}}=k \sqrt{\rho}, \\
& \left(\frac{s}{\sqrt{\rho}}\right)^{\prime}+\frac{1}{2} \frac{s^{2}}{\sqrt{\rho}}=k \sqrt{\rho} .
\end{aligned}
$$

\section{Global smooth solutions for subcritical initial data}

For the pressure-less Euler-Poisson equations (1.2), the evolution of $u_{x}$ and $\rho$ could be traced backwards along the same particle path to their initial data at $t=0$. The scenario becomes more complicated with the additional pressure term, due to the coupling of $r$ and $s$ along different particle paths which are traced back to different neighborhoods of the initial line $t=0$. This is the main obstacle in finding the sharp critical threshold of the full Euler-Poisson system (1.4). To this end, we will seek invariant regions for the coupled system, governing the Riemann invariants. We begin this section with the following lemma.

Lemma 3.1. If the total charge $E_{0}:=\int_{-\infty}^{\infty} \rho_{0}(x) d x$ is finite, then $\rho(x, t)$ and $u(x, t)$ remain uniformly bounded for all $t>0$.

Proof. Under the given condition, we can set (e.g., [ELT01, p. 116])

$$
\varphi_{x}(x, t)=\frac{1}{2}\left(\int_{-\infty}^{x} \rho(\xi, t) d \xi-\int_{x}^{\infty} \rho(\xi, t) d \xi\right),
$$

which satisfies $-E_{0} \leq \varphi_{x}(x, t) \leq E_{0}$ for all $t \geq 0$ and $x \in \mathbb{R}$.

Recall the transport equations (2.3a), 2.3b which govern the Riemann invariants along different characteristics $R^{`}+k \varphi_{x}=S^{\prime}+k \varphi_{x}=0$. Since $\varphi_{x}$ is bounded, these transport equations tell us that $R$ and $S$ remain uniformly bounded with at most a linear growth in time. Indeed, for all $M \gg 1$ we have

$$
\sup _{|x| \leq M}\{|R(x, t)|,|S(x, t)|\} \leq C_{0}+k E_{0} t, \quad C_{0}:=\sup _{|x| \leq M+u_{\infty} t}\left\{\left|R_{0}(x)\right|,\left|S_{0}(x)\right|\right\} .
$$

Take the sum and difference of $S$ and $R$ to find that $u(x, t)$ and $\rho(x, t)$ in (2.2) remain bounded, namely, there exists a constant (depending on $1 / A$ ) such that

$$
\begin{aligned}
& u_{\infty}:=\sup _{|x| \leq M}|u(x, t)| \leq C_{0}+k E_{0} t, \\
& \sup _{|x| \leq M} \rho(x, t) \leq \mathrm{const} \cdot \begin{cases}\left(C_{0}+k E_{0} t\right)^{2 /(\gamma-1)}, & \gamma>1, \\
\exp \left(k E_{0} t\right), & \gamma=1 .\end{cases}
\end{aligned}
$$


We note in passing that the time growth asserted in 3.2 is probably not sharp; the estimate can be improved after taking into account the uniform bounds of $R_{x} / \sqrt{\rho}$ and $S_{x} / \sqrt{\rho}$ discussed in Theorems 3.1 and 3.2 below.

Remark 3.1. According to [ELT01, Theorem 2.2], the breakdown of the "pressure-less" Euler-Poisson equations (1.2) occurs when $-u_{x}(x, t)$ and $\rho(x, t)$ approach $+\infty$ simultaneously at the critical time, $t \uparrow t_{c}$. In contrast, Lemma 3.1 tells us that the addition of pressure prevents the concentration of the density. Thus, the only breakdown for the full Euler-Poisson system (1.4) occurs through the formation of shock discontinuities, where $\left|u_{x}\right|$ and/or $\left|\rho_{x}\right|$ blow up as $t \uparrow t_{c}$, but neither $u$ nor $\rho$ will concentrate at any critical point.

\subsection{Critical threshold for isothermal case: $\gamma=1$}

We begin with the isothermal case, $\gamma=1$, which plays an important role in various applications. Compared with the general case 2.8), the isothermal case becomes simpler due to the fact that $\theta=0$ decouples the dependence on $r$ and $s$ through the mixed term $\theta r s$, which disappears from the left hand side of 2.9. Here we prove the following sharp characterization of the critical threshold phenomena.

Theorem 3.1. Consider the isothermal Euler-Poisson system (1.4) with pressure forcing $p(\rho)=A \rho$, and subject to initial data $u_{0}, \rho_{0}>0$ with finite total charge, $E_{0}=$ $\int_{-\infty}^{\infty} \rho_{0}(x) d x<\infty$. The system admits a global $C^{1}$-smooth solution if and only if

$$
u_{0 x}(x) \geq-\sqrt{2 k \rho_{0}(x)}+\sqrt{A} \frac{\left|\rho_{0 x}(x)\right|}{\rho_{0}(x)}, \quad \forall x \in \mathbb{R} .
$$

Remark 3.2. Expressed in terms of the Riemann invariants $u_{x} \pm \sqrt{A} \rho_{x} / \rho$, specified in $\$ 2.2$ Theorem 3.1 states that the isothermal Euler-Poisson equations admit global smooth solutions for subcritical initial conditions,

$$
s_{0} \geq-\sqrt{2 k \rho_{0}} \text { and } r_{0} \geq-\sqrt{2 k \rho_{0}} .
$$

Proof of Theorem 3.1. We define $X:=r / \sqrt{\rho}$ and $Y:=s / \sqrt{\rho}$. Equations 2.9a, 2.9b) then read

$$
\begin{aligned}
& X^{\prime}=\frac{\sqrt{\rho}}{2}\left(2 k-X^{2}\right), \\
& Y^{\prime}=\frac{\sqrt{\rho}}{2}\left(2 k-Y^{2}\right) .
\end{aligned}
$$

It follows that

$$
X^{\backslash} \begin{cases}>0, & X \in(-\sqrt{2 k}, \sqrt{2 k}) \\ =0, & |X|=\sqrt{2 k} \\ <0, & |X|>\sqrt{2 k}\end{cases}
$$


and similarly,

$$
Y^{\prime} \begin{cases}>0, & Y \in(-\sqrt{2 k}, \sqrt{2 k}) \\ =0, & |Y|=\sqrt{2 k} \\ <0, & |Y|>\sqrt{2 k}\end{cases}
$$

Thus, starting with 3.4 $, X_{0}, Y_{0} \geq-\sqrt{2 k}$, we find that $X$ and $Y$ remain bounded within the invariant region $[-\sqrt{2 k}, \sqrt{2 k}]$, or otherwise, they are decreasing outside this interval. We conclude that

$$
X(\cdot, t), Y(\cdot, t) \leq \max \left\{\sqrt{2 k}, X_{0}(\cdot), Y_{0}(\cdot)\right\} .
$$

Lemma 3.1 tells us that $\rho$ is bounded. The boundedness of $X, Y$ and $\rho$ implies that $r=X \sqrt{\rho}$ and $s=Y \sqrt{\rho}$ remain bounded for all $t<\infty$, and hence the Euler-Poisson system 1.4 admits a global $C^{1}$-smooth solution.

Conversely, suppose that there exists $X_{0}=X\left(x_{0}\right)<-\sqrt{2 k}$. We will show that this value will evolve along $\Gamma_{\lambda}\left(x_{0}, 0\right)$ so that $X(\cdot, t)$ will tend to $-\infty$ at a finite time. To this end, assume that $Y$ is well behaved, i.e., $Y_{0}(\cdot) \geq-\sqrt{2 k}$ so that $Y(\cdot, t) \leq Y_{1}:=$ $\max \left\{Y_{0}(\cdot), \sqrt{2 k}\right\}$ for all $t$ 's (otherwise, the finite time blow up of $Y$ can be argued along the same lines). It follows that $s=Y \sqrt{\rho} \leq Y_{1} \sqrt{\rho}$ and inserting this into $\rho^{\prime}=-\rho s$, we find $\rho^{\prime} \geq-Y_{1} \rho^{3 / 2}$. This yields the lower bound

$$
\rho \geq\left(\frac{2}{Y_{1} t+2 / \sqrt{\rho_{0}}}\right)^{2},
$$

and together with $3.5 \mathrm{a}$, we conclude that $X(\cdot, t)$ satisfies the following Riccati inequality along the $\Gamma_{\lambda}$-path:

$$
X^{\prime} \leq-\frac{X_{1}}{Y_{1} t+2 / \sqrt{\rho_{0}}} X^{2}, \quad X_{1}:=\left(X_{0}^{2}-2 k\right) / X_{0}^{2}>0 .
$$

Integration of 3.6 yields

$$
X(\cdot, t) \leq \frac{Y_{1}}{X_{1} \ln \left(1+\sqrt{\rho_{0}} Y_{1} t / 2\right)+Y_{1} X_{0}} .
$$

Thus, starting with $X_{0}<-\sqrt{2 k}<0$ it follows that there exists a finite critical time $t_{c}>0$ such that $X\left(t \uparrow t_{c}\right)$ tends to $-\infty$.

The critical threshold condition (3.3) reflects the competition between the Poisson forcing and the pressure. It yields global smooth solutions for a "large" set of initial configurations allowing negative velocity gradients. In the particular case where there is no pressure, $A=0, \sqrt{3.3}$ is reduced to the critical threshold condition of the "pressure-less" EulerPoisson equations, $u_{0 x}>-\sqrt{2 k \rho_{0}(x)}$, of [ELT01]. 


\subsection{Critical threshold for $\gamma>1$}

The equations for the Riemann invariants 2.8a, 2.8b are coupled through the mixed term, $\theta r s / 2$. We note in passing that it is possible to get rid of this mixed term when integrating 2.7a), 2.7b with the integration factors $\rho^{(\gamma-3) / 4}$ and $r \rho^{(\gamma-7) / 4}(3-\gamma) / 4$ in the first and second equations in each pair, yielding

$$
\begin{aligned}
& \left(r \rho^{(\theta-1) / 2}\right)^{\prime}+\frac{1+\theta}{2} r^{2} \rho^{(\theta-1) / 2}=k \rho^{(1+\theta) / 2}, \\
& \left(s \rho^{(\theta-1) / 2}\right)^{\prime}+\frac{1+\theta}{2} s^{2} \rho^{(\theta-1) / 2}=k \rho^{(1+\theta) / 2} .
\end{aligned}
$$

Nevertheless, it will prove useful to use the same integration factors, $1 / \sqrt{\rho}$ and $r / 2 \rho \sqrt{\rho}$, which led to 2.8). The main task is to identify the invariant region associated with (2.8), corresponding to the isothermal invariant region $[-\sqrt{2 k}, \sqrt{2 k}]$ discussed in Theorem 3.1

Theorem 3.2. Consider the Euler-Poisson system (1.4) with $\gamma$-law pressure $p(\rho)=$ $A \rho^{\gamma}, \gamma>1$, subject to initial data $u_{0}, \rho_{0}>0$ with finite total charge, $E_{0}=\int_{-\infty}^{\infty} \rho_{0}(x) d x$ $<\infty$. Then there exists a constant $K_{0}>0$, depending on $k, \gamma$ and the initial conditions (specified in (3.9b below), such that the Euler-Poisson equations (1.4) admit a global $C^{1}$-smooth solution if

$$
u_{0 x}(x) \geq-K_{0} \sqrt{\rho_{0}(x)}+\sqrt{A \gamma} \frac{\left|\rho_{0 x}(x)\right|}{\rho_{0}(x)^{(3-\gamma) / 2}} .
$$

Before we turn to the proof of this theorem, several remarks are in order.

Remark 3.3. Expressed in terms of the Riemann invariants, $r=u_{x}-\sqrt{A \gamma} \rho_{0 x} / \rho_{0}^{(3-\gamma) / 2}$ and $s=u_{x}+\sqrt{A \gamma} \rho_{0 x} / \rho_{0}^{(3-\gamma) / 2}$, the critical threshold 3.8 reads

$$
\frac{r_{0}(x)}{\sqrt{\rho_{0}(x)}}, \frac{s_{0}(x)}{\sqrt{\rho_{0}(x)}} \geq-K_{0} .
$$

The constant $K_{0}$ is given by

$$
K_{0}=\frac{-\theta M_{0}+\sqrt{\theta^{2} M_{0}^{2}+8 k(1+\theta)}}{2(1+\theta)}, \quad M_{0}=\max _{x}\left\{\sqrt{2 k}, \frac{r_{0}(x)}{\sqrt{\rho_{0}(x)}}, \frac{s_{0}(x)}{\sqrt{\rho_{0}(x)}}\right\} \text {. }
$$

We mention two simplifications which are summarized in the following two corollaries. We first observe that if the initial configurations satisfy the upper bound $r_{0}(x), s_{0}(x)$ $\leq \sqrt{2 k \rho_{0}(x)}$ then $3.9 \mathrm{~b}$ yields $M_{0}=\sqrt{2 k}$, hence $K_{0}=\sqrt{2 k} /(1+\theta)$, and Theorem 3.2 implies the following. 
Corollary 3.1. Consider the Euler-Poisson system 1.4 with $p(\rho)=A \rho^{\gamma}, \gamma>1$, subject to initial data $u_{0}, \rho_{0}>0$ with $E_{0}=\int_{-\infty}^{\infty} \rho_{0}(x) d x<\infty$. Then the Euler-Poisson equations 1.4 admit a global $C^{1}$-smooth solution if for all $x \in \mathbb{R}$,

$$
\left|u_{0 x}(x)\right| \leq \frac{\sqrt{2 k \rho_{0}(x)}}{1+\theta}-\sqrt{A \gamma} \frac{\left|\rho_{0 x}(x)\right|}{\rho_{0}(x)^{(3-\gamma) / 2}} .
$$

The next result follows from the trivial inequality

$$
-K_{0} \leq \frac{\theta M_{0}-\left(\theta M_{0}+\sqrt{8 k(1+\theta)}\right) / \sqrt{2}}{2(1+\theta)} .
$$

Corollary 3.2. Consider the Euler-Poisson system (1.4) with $p(\rho)=A \rho^{\gamma}, \gamma>1$, subject to initial data $u_{0}, \rho_{0}>0$ with $E_{0}=\int_{-\infty}^{\infty} \rho_{0}(x) d x<\infty$. Then the Euler-Poisson equations 1.4 admit a global $C^{1}$-smooth solution if for all $x \in \mathbb{R}$,

$$
\begin{aligned}
u_{0 x}(x) \geq & -\sqrt{\frac{2 k \rho_{0}(x)}{\gamma+1}} \\
& +\left(1-\frac{1}{\sqrt{2}}\right) \frac{\gamma-1}{2(\gamma+1)} \max _{x}\left\{\sqrt{2 k \rho_{0}(x)}, u_{0 x}(x)+\sqrt{A \gamma} \frac{\left|\rho_{0 x}(x)\right|}{\rho_{0}(x)^{(3-\gamma) / 2}}\right\} \\
& +\sqrt{A \gamma} \frac{\left|\rho_{0 x}(x)\right|}{\rho_{0}(x)^{(3-\gamma) / 2}} .
\end{aligned}
$$

Remark 3.4. We observe that as in the isothermal case, the critical threshold in its various versions (3.8)- (3.11) allows a "large" set of initial configurations with negative velocity gradient, due to the competition between the stabilizing Poisson forcing $k \rho \phi(\rho)_{x}$ and the destabilizing pressure $A\left(\rho^{\gamma}\right)_{x}$. In the extreme case where Poisson forcing is missing, $k=0$, the breakdown of the system is generic unless $u_{0 x}$ is positive enough (so that $r_{0}, s_{0}>0$ ). In the other extreme of a "pressure-less" Euler-Poisson, $A=0, \gamma=1$, the critical thresholds $\sqrt{3.8}$, 3.10 are reduced to $u_{0 x}(x)>-\sqrt{2 k \rho_{0}(x)}$, which coincides with the "pressure-less" critical threshold (1.3) found in [ELT01].

Proof of Theorem 3.2 Expressed in terms of $X:=r / \sqrt{\rho}$ and $Y:=s / \sqrt{\rho}$, equations (2.8) read

$$
\begin{aligned}
& X^{\prime}=\sqrt{\rho}\left(k-\frac{1+\theta}{2} X^{2}+\frac{\theta}{2} X Y\right), \\
& Y^{\prime}=\sqrt{\rho}\left(k-\frac{1+\theta}{2} Y^{2}+\frac{\theta}{2} X Y\right) .
\end{aligned}
$$

We seek an invariant region of the form $\left[-K_{0}, M_{0}\right]$, with $K_{0}, M_{0}>0$ yet to be determined. To this end we construct a "buffer zone" in which positive values of $X, Y$ must 
decrease and hence remain upper bounded. We begin by noticing that if $X, Y \leq M$ then 1 $X_{+} Y \leq M^{2}$, and recalling that $\theta \geq 0,3.12$ then yields

$$
\begin{array}{rlrl}
X^{\prime} & \leq \sqrt{\rho}\left(k-\frac{1+\theta}{2} X^{2}+\frac{\theta}{2} M^{2}\right), & X>0, \\
Y^{\prime} \leq \sqrt{\rho}\left(k-\frac{1+\theta}{2} Y^{2}+\frac{\theta}{2} M^{2}\right), & Y>0 .
\end{array}
$$

This in turn implies that

$X$ resp. $Y$ is decreasing whenever $X \in \mathcal{I}_{M}$ resp. $Y \in \mathcal{I}_{M}$.

Here $\mathcal{I}_{M}$ is the interval $\left(C_{+}(M), M\right)$ where $C_{+}(M):=\sqrt{\left(2 k+\theta M^{2}\right) /(1+\theta)}$ is dictated by the largest root of the quadratics on the right of $(3.13)$. Observe that for $\mathcal{I}_{M}$ to be nonempty we need $M>\sqrt{2 k}$. We therefore set $M_{\epsilon}:=\max _{x}\left\{\sqrt{2 k}+\epsilon, X_{0}(x), Y_{0}(x)\right\}$. We claim that $X=X(\cdot, t)$ and $Y=Y(\cdot, t)$ remain bounded at later time, $X, Y \leq M_{\epsilon}$ : indeed, either $X, Y \leq C_{+}\left(M_{\epsilon}\right)<M_{\epsilon}$ or, if $X, Y>C_{+}\left(M_{\epsilon}\right)$, then they must decrease being "trapped" inside $\mathcal{I}_{M_{\epsilon}}$ and hence $X, Y \leq M_{\epsilon}$. Letting $\epsilon \downarrow 0$ we end up with the upper bound

$$
X(\cdot, t), Y(\cdot, t) \leq M_{0}, \quad M_{0}:=\max _{x}\left\{\sqrt{2 k}, X_{0}(x), Y_{0}(x)\right\}
$$

In a similar manner, we study the lower bound of the invariant region. (3.14) and 3.12 yield

$$
\begin{array}{ll}
X^{\prime} \geq \sqrt{\rho}\left(k-\frac{1+\theta}{2} X^{2}+\frac{\theta}{2} M_{0} X\right), & X<0, \\
Y^{\prime} \geq \sqrt{\rho}\left(k-\frac{1+\theta}{2} Y^{2}+\frac{\theta}{2} M_{0} Y\right), & Y<0,
\end{array}
$$

which in turn imply that

$$
X \text { and } Y \text { are increasing if } 0 \geq X, Y>-K_{0},
$$

where $K_{0}$ is the smallest root of the quadratics on the right of 3.15 ,

$$
K_{0}:=\frac{-\theta M_{0}+\sqrt{\theta^{2} M_{0}^{2}+8 k(1+\theta)}}{2(1+\theta)} .
$$

The critical threshold condition (3.8) tells us that at $t=0, X_{0}, Y_{0} \geq-K_{0}$, and 3.16a) implies that $X(\cdot, t)$ and $Y(\cdot, t)$ remain above the same lower bound, (3.8). As before, the bounds of $X, Y$ and $\rho$ imply that $r=X \sqrt{\rho}$ and $s=Y \sqrt{\rho}$ remain bounded, and hence the Euler-Poisson system 1.4 a global $C^{1}$-smooth solution.

\footnotetext{
1 We let $Z_{+}=\max \{X, 0\}$ and $Z_{-}=\min \{Z, 0\}$ denote the positive and negative part of $Z$.
} 


\section{Finite-time breakdown for supercritical initial data}

Consider the Euler-Poisson system 1.4 with a $\gamma$-law pressure, $\gamma \geq 1$, and subject to initial data such that $r_{0}(x), s_{0}(x) \leq \sqrt{2 k}$. Then, according to Corollary 3.1 the following critical threshold is sufficient for the existence of global smooth solutions:

$$
u_{0 x}(x) \geq-\sqrt{2 k \rho_{0}(x)}+\sqrt{A \gamma} \frac{\left|\rho_{0 x}(x)\right|}{\rho_{0}(x)^{(3-\gamma) / 2}} .
$$

In this section we show that this critical threshold is also necessary for global regularity.

Theorem 4.1. Consider the Euler-Poisson system (1.4) with $\gamma$-law pressure $p(\rho)=$ $A \rho^{\gamma}, \gamma \geq 1$, subject to initial data $u_{0}, \rho_{0}>0$. The system loses the $C^{1}$-smoothness if there exists an $x \in \mathbb{R}$ such that

$$
u_{0 x}(x)<-\sqrt{2 k \rho_{0}(x)}+\sqrt{A \gamma} \frac{\left|\rho_{0 x}(x)\right|}{\rho_{0}(x)^{(3-\gamma) / 2}} .
$$

Remark 4.1. Expressed in terms of the Riemann invariants, $r=u_{x}-\sqrt{A \gamma} \rho_{0 x} / \rho_{0}^{(3-\gamma) / 2}$ and $s=u_{x}+\sqrt{A \gamma} \rho_{0 x} / \rho_{0}^{(3-\gamma) / 2}$, the condition 4.1 reads

$$
\exists x \in \mathbb{R} \quad r_{0}(x)<-\sqrt{2 k \rho_{0}(x)} \quad \text { or } \quad s_{0}(x)<-\sqrt{2 k \rho_{0}(x)} .
$$

The lack of smoothness in this case was shown in Theorem 3.1 for $\gamma=1$ and is extended for $\gamma>1$ below.

Proof of Theorem 4.1. Recall equations 3.12 for $X:=r / \sqrt{\rho}$ and $Y:=s / \sqrt{\rho}$,

$$
\begin{aligned}
& X^{\prime}=\sqrt{\rho}\left(k-\frac{1+\theta}{2} X^{2}+\frac{\theta}{2} X Y\right), \\
& Y^{\prime}=\sqrt{\rho}\left(k-\frac{1+\theta}{2} Y^{2}+\frac{\theta}{2} X Y\right) .
\end{aligned}
$$

In the proof of Theorem 3.2, we have shown that $X$ and $Y$ have an upper bound

$$
X(\cdot, t), Y(\cdot, t) \leq M_{0}, \quad M_{0}:=\max _{x}\left\{\sqrt{2 k}, X_{0}(x), Y_{0}(x)\right\}
$$

Suppose that there exists $X_{0}=X\left(x_{0}\right)<-\sqrt{2 k}$. We will show that this value will evolve along $\Gamma_{\lambda}\left(x_{0}, 0\right)$ in such a way that $X(\cdot, t)$ will tend to $-\infty$ at a finite time. To this end, assume that $Y$ is well behaved, i.e., $Y_{0}(\cdot) \geq-\sqrt{2 k}$ so that $Y(\cdot, t) \leq M_{0}$ for all $t$ 's (otherwise, the finite time blow up of $Y$ can be deduced along the same lines). It follows that along $\Gamma_{\lambda}\left(x_{0}, 0\right)$,

$$
X^{\prime}=\sqrt{\rho}\left(k-\frac{1+\theta}{2} X^{2}+\frac{\theta}{2} X Y\right)<\sqrt{\rho}\left(k-\frac{1}{2} X^{2}\right) .
$$


Following exactly what we have done in the proof of Theorem 3.1 , we obtain the inequality

$$
X(\cdot, t) \leq \frac{M_{0}}{X_{1} \ln \left(1+\sqrt{\rho_{0}} M_{0} t / 2\right)+M_{0} X_{0}},
$$

where $X_{1}:=\left(X_{0}^{2}-2 k\right) / X_{0}^{2}>0$. Thus, starting with $X_{0}<-\sqrt{2 k}<0$ it follows that there exists a finite critical time $t_{c}>0$ such that $X\left(t \uparrow t_{c}\right)$ tends to $-\infty$.

We conclude with an example for a finite-time breakdown.

Example. Suppose at $t=0, u_{0}(x)=0$ and

$$
\rho_{0}(x)= \begin{cases}1, & x<0 \\ 1-x / 2 \epsilon, & 0 \leq x \leq \epsilon, \\ 1 / 2, & x>\epsilon\end{cases}
$$

Thus

$$
s_{0}(x)= \begin{cases}-\sqrt{A \gamma}(1-x / 2 \epsilon) / 2 \epsilon, & 0<x<\epsilon, \\ 0, & \text { elsewhere. }\end{cases}
$$

If we choose $\epsilon$ small enough, then $s_{0}(x)<-\sqrt{2 k \rho_{0}(x)}$ for $0<x<\epsilon$. According to Theorem 4.1 the system 1.4 will break down in a finite time. This example shows that even if the fluid is near rest at $t=0$, the pressure itself could still lead to collision.

Acknowledgments. This research was supported in part by NSF grant 04-07704 and by ONR grant N00014-91-J-1076.

\section{References}

[BW98] Brenner, M. P., Witelski, T. P.: On spherically symmetric gravitational collapse. J. Statist. Phys. 93, 863-900 (1998) Zbl 0933.35160 MR 1666522

[CW96] Chen, G.-Q., Wang, D.: Convergence of shock capturing scheme for the compressible Euler-Poisson equation. Comm. Math. Phys. 179, 333-364 (1996) Zbl 0858.76051 MR 1400743

[En96] Engelberg, S.: Formation of singularities in the Euler and Euler-Poisson equations. Phys. D 98, 67-74 (1996) Zbl 0885.35086 MR 1416291

[ELT01] Engelberg, S., Liu, H., Tadmor, E.: Critical thresholds in Euler-Poisson equations. Indiana Univ. Math. J. 50, 109-157 (2001) Zbl 0989.35110 MR 1855666

[Gu98] Guo, Y.: Smooth irrotational flows in the large to the Euler-Poisson system in $\mathbb{R}^{3+1}$. Comm. Math. Phys. 195, 249-265 (1998) Zbl 0929.35112 MR 1637856

[JR00] Junca, S., Rascle, M.: Relaxation of the isothermal Euler-Poisson system to the drift-diffusion equations. Quart. Appl. Math. 58, 511-521 (2000) Zbl 02051300 MR 1770652

[La64] Lax, P. D.: Development of singularities of solutions of nonlinear hyperbolic partial differential equations. J. Math. Phys. 5, 611-613 (1964) Zbl $0135.15101 \mid$ MR 0165243

[LT02] Liu, H., Tadmor, E., Spectral dynamics of the velocity gradient field in restricted flow. Comm. Math. Phys. 228, 435-466 (2002) Zbl 1031.76006 MR 1918784

[LT03] Liu, H., Tadmor, E.: Critical thresholds in 2D restricted Euler-Poisson equations. SIAM J. Appl. Math. 63, 1889-1910 (2003) Zbl 1073.35187 MR 2030849 
[Ma86] Makino, T.: On a local existence theorem for the evolution of gaseous stars. In: Patterns and Waves, T. Nishida et al. (eds.), North-Holland/Kinokuniya, 459-479 (1986) Zbl 0623.35058 MR 0882389

[MP90] Makino, T., Perthame, B.: Sur les solutions à symétrie sphérique de l'équation d'EulerPoisson pour l'évolution d'étoiles gazeuses. Japan J. Appl. Math. 7, 165-170 (1990) Zbl 0743.35048 MR 1039243

[MN95] Marcati, P., Natalini, R.: Weak solutions to a hydrodynamic model for semiconductors and relaxation to the drift-diffusion equation. Arch. Ration. Mech. Anal. 129, 129-145 (1995) Zbl 0829.35128 MR 1328473

[Pe90] Perthame, B.: Nonexistence of global solutions to the Euler-Poisson equations for repulsive forces. Japan J. Appl. Math. 7, 363-367 (1990) Zbl 0717.35049 MR 1057537

[PRV95] Poupaud, F., Rascle, M., Vila, J.-P.: Global solutions to the isothermal Euler-Poisson system with arbitrarily large data. J. Differential Equations 123, 93-121 (1995) Zbl 0845.35123 MR 1359913

[Si85] Sideris, T. C.: Formation of singularities in three-dimensional compressible fluids. Comm. Math. Phys. 101, 475-485 (1985) Zbl 0606.76088 MR 0815196

[WC98] Wang, D., Chen, G.-Q.: Formation of singularities in compressible Euler-Poisson fluids with heat diffusion and damping relaxation. J. Differential Equations 144, 44-65 (1998) Zbl 0914.35102 MR 1615127 